Original paper

\title{
Effects of shielding on pelvic and abdominal IORT dose distributions
}

\author{
Alessandro Esposito ${ }^{a}$, Taxiarchis Sakellaris ${ }^{a}$, Patrícia Limede ${ }^{a}$, Filipa Costa ${ }^{\mathrm{b}}$, Luis T. Cunha ${ }^{\mathrm{b}, \mathrm{c}}$, \\ Anabela Gregório Dias ${ }^{\mathrm{b}, \mathrm{c}}$, Joana Lencart ${ }^{\mathrm{b}, \mathrm{c}}$, Sandra Sarmento ${ }^{\mathrm{b}, \mathrm{c}}$, Carla Carmelo Rosa ${ }^{\mathrm{a}, \mathrm{d}, *}$ \\ a INESC TEC - INESC Technology and Science, Porto, Portugal \\ ${ }^{\mathrm{b}}$ Medical Physics, Radiobiology and Radiation Protection Group, IPO Porto Research Centre (CI-IPOP), Portuguese Oncology Institute of Porto (IPO Porto), Porto, Portugal \\ ${ }^{\mathrm{c}}$ Medical Physics Department, Portuguese Oncology Institute of Porto (IPO Porto), Porto, Portugal \\ ${ }^{\mathrm{d}}$ Departamento de Física e Astronomia, Faculdade de Ciências, Universidade do Porto, Porto, Portugal
}

\section{A R T I C L E I N F O}

\section{Article history:}

Received 24 May 2016

Received in Revised form 4 August 2016

Accepted 5 October 2016

Available online 22 October 2016

\section{Keywords:}

Monte Carlo simulations

Pelvic IORT

Shielding

Dose distributions

\begin{abstract}
A B S T R A C T
Purpose: To study the impact of shielding elements in the proximity of Intra-Operative Radiation Therapy (IORT) irradiation fields, and to generate graphical and quantitative information to assist radiation oncologists in the design of optimal shielding during pelvic and abdominal IORT.

Method: An IORT system was modeled with BEAMnrc and EGS++ Monte Carlo codes. The model was validated in reference conditions by gamma index analysis against an experimental data set of different beam energies, applicator diameters, and bevel angles. The reliability of the IORT model was further tested considering shielding layers inserted in the radiation beam. Further simulations were performed introducing a bone-like layer embedded in the water phantom. The dose distributions were calculated as 3D dose maps.

Results: The analysis of the resulting 2D dose maps parallel to the clinical axis shows that the bevel angle of the applicator and its position relative to the shielding have a major influence on the dose distribution. When insufficient shielding is used, a hotspot nearby the shield appears near the surface. At greater depths, lateral scatter limits the dose reduction attainable with shielding, although the presence of bone-like structures in the phantom reduces the impact of this effect.

Conclusions: Dose distributions in shielded IORT procedures are affected by distinct contributions when considering the regions near the shielding and deeper in tissue: insufficient shielding may lead to residual dose and hotspots, and the scattering effects may enlarge the beam in depth. These effects must be carefully considered when planning an IORT treatment with shielding.
\end{abstract}

(c) 2016 Associazione Italiana di Fisica Medica. Published by Elsevier Ltd. All rights reserved.

\section{Introduction}

During a surgical intervention, after tumor resection, ionizing radiation is sometimes used for direct treatment of the resection margins, while nearby sensitive tissues are moved away or shielded from the treatment beam [1,2]. High-energy electron beams are frequently used for this purpose due to their relatively low penetration depth, when compared to high energy photons, allowing the sparing of healthy organs underneath. This technique is known as Intra-Operative Electron Radiation Therapy (IORT). At our Institution IORT is used almost exclusively to treat locally advanced or recurrent rectal cancer, which is treated with preoperative radiochemotherapy followed by surgery with IORT. IORT is not a new technique, but its high cost initially hindered its

\footnotetext{
* Corresponding author at: CAP/INESC-Tec, R. do Campo Alegre 687, 4169-007 Porto, Portugal.

E-mail address: ccrosa@fc.up.pt (C.C. Rosa).
}

development. Recently, the more affordable option of mobile linear accelerators led to a renewed interest in the technique [3].

In abdominal and pelvic IORT there is an occasional need to use small shielding elements to partially block the radiation field (beam shaping) due to irregularities in the shape of the tumour, and the occasional presence of adjacent sensitive tissues which cannot be moved out of the beam path [4,5]. The ideal shielding should be quick to mould at the IORT stage, easily adaptable to the treatment scenario, and thin enough not to interfere with the position of the applicator. Lead $(\mathrm{Pb})$ is obviously a good option to fulfill these requirements. Sterilized thin sheets of lead, wrapped in gauze and soaked in saline solution to avert lead-tissue contact, may be cut to the appropriate shape and inserted in the patient, in sufficient number to reduce the dose down to the level of the Bremsstrahlung tail. According to Biggs et al., the effect of this field shaping on the output factors is small $(<2-3 \%)$ if the amount of field blocking is not very large $(<25 \%)$ [5]. Bagne et al. recommend using sufficient lead to reduce the dose in the blocked area to less 
than $10 \%$ of the unblocked dose, and indicate approximate values of lead thickness, stressing that these must be verified for each individual machine before clinical use [4].

The indications of Bagne et al. were not accompanied by graphical representations of dose distributions. However, the visualization of dose distributions is very important in IORT, since the volume to be irradiated is visually estimated at the operating theatre by the radiation oncologist, who chooses the electron energy according to the depth of tissue to be treated, based on the isodose curves measured under reference conditions. Dose calculations are usually done manually, using tables based on water tank measurements, because planning software would require a CT scan [6] and this is difficult to obtain at the surgical stage.

Monte Carlo (MC) simulations are often used to study the effects of shielding without the difficulties associated with experimental measurement [7], allowing 3D dose maps analysis. Upon validation in standard geometries, such as water phantoms, MC is a good predictor in the study of different geometries. Monte Carlo simulations have been used to optimize the composition of breast IORT shielding disks, to obtain adequate protection of the organs beneath the shielding, ensuring the prescribed dose to the irradiated volume [8-11]. This optimization is specific to the irradiation geometry of breast IORT, where the irradiation surface is nearly flat and easily accessible, the shielding is placed under the tissue to be irradiated (disc-shaped shielding elements from a few millimetres up to more than $1 \mathrm{~cm}$ can be used), and the IORT applicators are usually circular and non-beveled. Soriani et al. also used Monte Carlo (MC) simulations to help design a beam-shaper device to transform a circular IORT beam $10 \mathrm{~cm}$ in diameter into a rectangular field of variable shape, in order to treat the elongated shapes typical of soft-tissue sarcomas in distal limbs [12]. The beam-shaper of Soriani et al. does not fit into the patient, therefore it is not applicable in pelvic or abdominal IORT, where the area to be irradiated is deep inside the surgical cavity.

In pelvic (e.g. rectum) and abdominal (e.g. pancreas) IORT the typical irradiation geometries are completely different from those of breast and soft-tissue sarcomas, as are the typical field shapes. The relative location of organs at risk and the accessibility of the irradiation surface are also different. In addition, beveled applicators are often necessary, and shielding requirements vary according to the clinical situation. Therefore, it seems important to have a complete and detailed characterization of shielding effects on the dose distribution, complemented with graphical analysis, to serve as a guideline for professionals working on the field.

The aim of this study is to characterize the effect of lead shielding in typical scenarios of pelvic and abdominal IORT, providing relevant and important information for radiation oncologists to optimize pelvic IORT. Monte Carlo simulations evaluate the effect of lead shielding on dose distributions with high level of detail and visualization which is only possible with computer simulations. The Monte Carlo IORT model is also explored in the study of bone influence to the dose distribution under irradiated and shielded tissue.

\section{Materials and methods}

\subsection{IORT system}

At the study location, IORT procedures are performed with a Varian CLINAC 2100CD conventional linear accelerator (LINAC), adapted for IORT with a system of hard-docking applicators. The LINAC is installed in a bunker adjacent to an Operating Room. It produces electron beams of five nominal energies $(6,9,12,15$ and $18 \mathrm{MeV}$ ) but only the lower four are clinically used. The IORT system includes polymethyl methacrylate (PMMA) cylindrical applicators of $3 \mathrm{~mm}$ wall thickness to which beveled PMMA terminations are attached through an intermediate brass joint (Fig. 1a)). Applicators are available with external diameters of $3-15 \mathrm{~cm}$, in steps of 1 or $2 \mathrm{~cm}$, and bevel angles of 0 (B0), 15 (B15), 30 (B30) and $45^{\circ}$ (B45). The most frequently used applicators are 6,7 and $8 \mathrm{~cm}$ in diameter, with bevel angles of 30 and $45^{\circ}$, at energies of 6, 9 and $12 \mathrm{MeV}$.

\subsection{IORT numeric model}

An accurate model of the IORT system was implemented using detailed LINAC specifications from the Varian Medical Systems Monte Carlo Data Package. The LINAC numerical model was implemented using BEAMnrc, an EGSnrc based MC code used for simulating photon and electron beams [13]. The MC model implementation was split into four simulation stages, producing four particle history phase-space files ( $p h s p$ ) along the LINAC and IORT structures.

The first two stages simulated the primary beam, producing phsp files just above (phsp1) and below (phsp2) the LINAC collimator jaws. The phsp2 was used in open-field simulations (without any IORT hardware attached to the LINAC head) to validate the LINAC numerical model, using the code DOSXYZnrc [14] to score the dose in a $60 \times 60 \times 10 \mathrm{~cm}^{3}$ (width $\times$ length $\times$ depth) water phantom with a $0.5 \times 0.5 \times 0.2 \mathrm{~cm}^{3}$ voxel size. Field sizes of $10 \times 10 \mathrm{~cm}^{2}$ and $40 \times 40 \mathrm{~cm}^{2}$ were analyzed. The collimator openings were chosen to match those of IORT procedures, in particular $20 \times 20 \mathrm{~cm}^{2}$ for 6 and $9 \mathrm{MeV}$, and $17 \times 17 \mathrm{~cm}^{2}$ for $12 \mathrm{MeV}$. The obtained numerical transverse dose profiles and percentage depth dose (PDD) curves were compared with experimental data acquired in similar configurations.

Following the previous validation, the IORT applicator system was implemented in two simulation stages. In the first, BEAMnrc was used to simulate the radiation field along the main applicator system (see Fig. 1a)), starting from the collimator output phsp2 and yielding in the final phase-space (phsp3) at the coupling external brass joint. The last simulation stage propagates phsp3 along the beveled applicator ending and the voxelized water phantom, both implemented in EGS++ code [15,16]. IORT applicators of 6,7 and $8 \mathrm{~cm}$ diameter of four possible bevel angles $\left(0,15,30\right.$ and $\left.45^{\circ}\right)$ were considered. The water phantom was redefined as a smaller $24 \times 24 \times 7 \mathrm{~cm}^{3}$ water tank at this stage of the simulation, considering that the maximum expected dimension of the radiation beams at the phantom surface is not greater than $8 \mathrm{~cm}$. A set of tests was performed to select the smallest possible voxel size that would give an acceptable statistical uncertainty within feasible simulation times. The criterion for an acceptable statistical uncertainty was: (i) for PDDs: an uncertainty of less than $1 \%$ for all depths from 0 down to the depth where the dose has fallen to $10 \%$ of the dose maximum at $\mathrm{d}_{\max }$ and (ii) for dose profiles: an uncertainty of less than $1 \%$ for the plateau region (90\%). Considering these criteria, the simulations were performed using $1.65 \times 10^{8}$ histories from phsp 3 with a $0.4 \times 0.4 \times 0.2 \mathrm{~cm}^{3}$ voxel size.

Finally, reference PDD curves and transverse dose profiles at various depths were simulated for all possible IORT configurations in water phantom, i.e. combinations of beam energy, applicator diameter, and bevel angle.

\subsection{IORT model validation}

Measurements were performed in a water tank (MP3-M, PTWFreiburg, Germany) with a diode detector (type 60012 E PTWFreiburg, Germany), for all simulated reference configurations. The validation PDD curves and transverse dose profiles were acquired using the measuring configuration recommended by the AAPM task group 48 (TG48) [2], where the "clinical axis" is defined 
a)

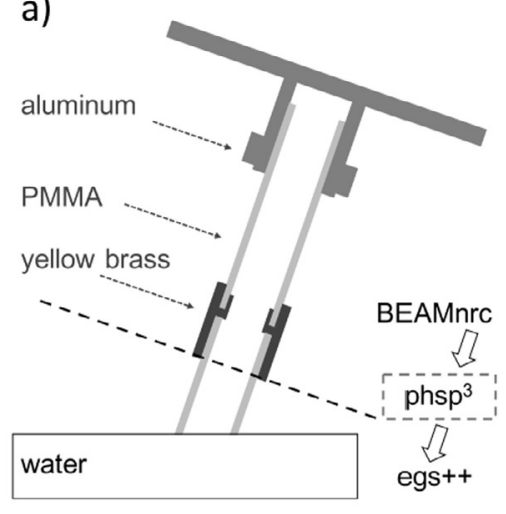

b)

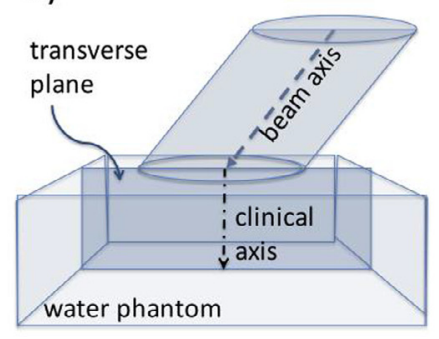

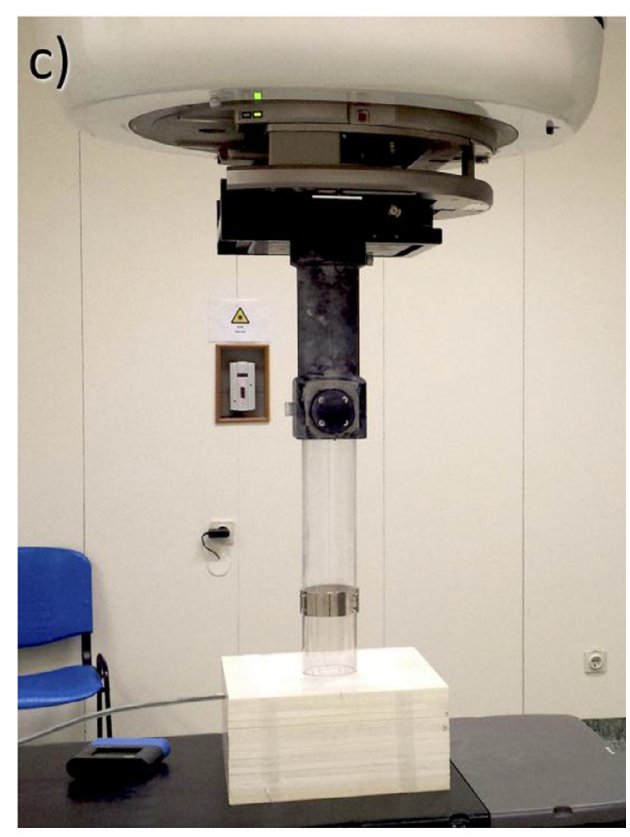

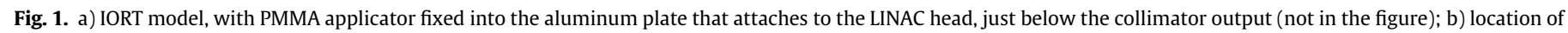
the transverse plane and clinical axis in the water phantom; c) photo of the IORT system with bevel $0^{\circ}$ applicator attached.

as the line perpendicular to the phantom surface intersecting the beam axis along the applicator at the surface (Fig. 1b)). According to TG48, the beam central axis of beveled applicators has little clinical relevance, since the vertical depth from the phantom surface is more relevant for IORT.

Global gamma index analysis [17], with $2 \%$ as dose difference and $2 \mathrm{~mm}$ as distance to agreement (DTA), was applied to assess the agreement between the simulated and the corresponding reference (measured) data for all combinations of beam energy, applicator diameter and bevel angle. Agreement is obtained when more than $95 \%$ of all evaluated points have a gamma index below 1 .

The reliability of the IORT model was further checked in nonreference conditions: the accuracy of the simulated dose distributions in water tank was assessed when highly attenuating pieces of shielding were introduced into the radiation field. Two $1.2 \mathrm{~mm}$ thick lead sheets were laterally inserted $1.6 \mathrm{~cm}$ in the radiation field of a B0 $7 \mathrm{~cm}$ applicator (7B0), corresponding to a field blocking of $17 \%$ of the applicator area. This shielding configuration was simulated for different beam energies, and dose profiles were measured in the same configuration, at several depths, with the lead sheets taped to the end of the applicator. The results were compared using gamma index analysis $(2 \%, 2 \mathrm{~mm})$.

\subsection{Studies on shielding in IORT}

Several IORT shielding configurations were studied using simulations to build a set of clinically relevant reference dose distributions for pelvic IORT.

The effects of using different total lead thicknesses, and the influence of the electron beam energy, are important factors to consider when a sensitive organ or tissue needs to be protected. The minimum lead thickness necessary to reduce the dose to less than $10 \%$ of the unshielded dose was determined from the IORT model simulations. Transverse dose profiles were extracted from the simulated 3D dose data mesh at 3 and $9 \mathrm{~mm}$ depths, for different energies and lead thicknesses. The considered lead thicknesses were integer multiples of the $1.2 \mathrm{~mm}$ clinically available lead sheets.

Color-coded dose distributions were created for different shielding configurations, beam energy and applicators, and visualized using the Computational Environment for Radiotherapy Research (CERR, version 3.1) [18]. All simulated dose distributions were normalized to the "clinical axis" dose maximum (Dmax) of the corresponding reference (i.e. the same applicator and energy in an unshielded configuration).

The presence of a shield between the applicator and the water phantom modifies the Dmax depending on the shield edge position. The shielded configuration can be derived from the reference setup in a two-step process. The first step displaces the applicator, determining an air gap, while the second inserts the shielding between the applicator and the water phantom. To distinguish the dosimetric changes due to the air gap and the shielding, the two effects were evaluated separately through specific simulations, with and without any shielding, for the three beam energies (6, 9 and $12 \mathrm{MeV}$ ).

Introducing shielding inside the patient during a surgery is not straightforward and several millimeters of lead are required for high beam energies. This is a considerable thickness to insert in the surgical cavity, especially since the lead must be wrapped in a sterile envelope and/or in gauze. Dose limits for sensitive tissues do not always require dose reduction to $10 \%$ of prescription, but current recommendations are to use sufficient thickness to reduce the dose down to the Bremsstrahlung tail level. Therefore, situations of insufficient shielding were simulated to allow a better understanding of its impact and of the rationale behind the recommendations.

In pelvic IORT for rectal cancer, the sacral bone is usually immediately below the irradiated area. Therefore, the effect of having bony structures under the tissue was also investigated and modeled through a non-homogeneous phantom, by simulating the insertion of a $3 \mathrm{~cm}$ layer of bone material, $5 \mathrm{~mm}$ beneath the phantom surface. 


\section{Results}

\subsection{IORT model validation}

Simulated PDDs and transverse dose profiles were compared with measured (reference) data using the gamma index model, with $2 \% / 2 \mathrm{~mm}$. Examples of agreement between simulated and

a)

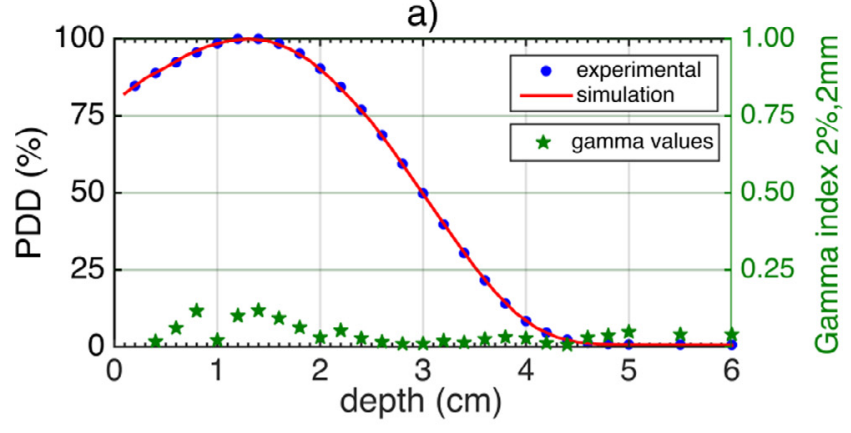

b)

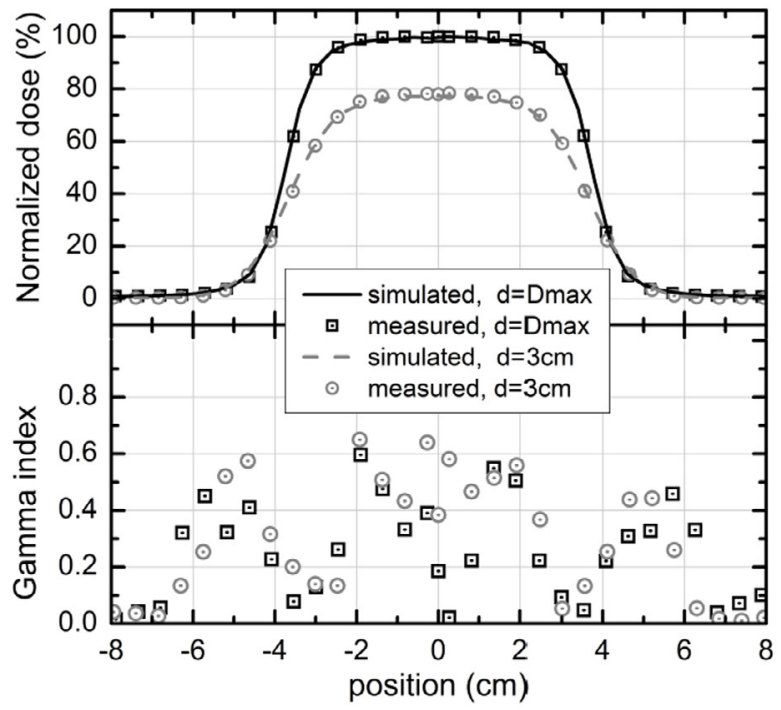

c)

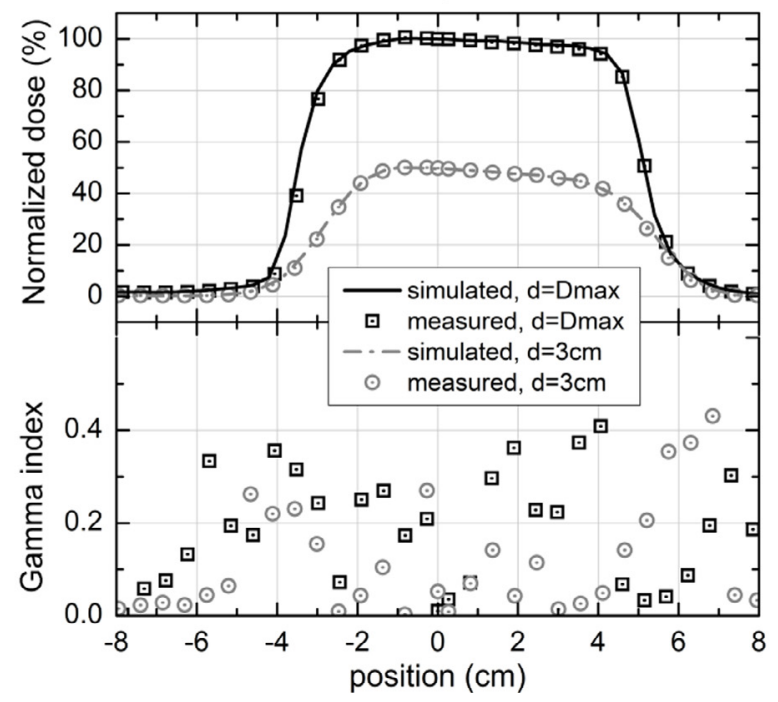

Fig. 2. Measured and simulated data for a $9 \mathrm{MeV}$ IORT beam and $8 \mathrm{~cm}$ applicator: (a) PDD profile for B30, with gamma index $(2 \% / 2 \mathrm{~mm})$ on the secondary axes; (b) dose profiles at $d_{\max }$ and $3.0 \mathrm{~cm}$ for $\mathrm{B} 0$; (c) dose profiles at $\mathrm{d}_{\max }$ and $3 \mathrm{~cm}$ for $\mathrm{B} 30$. experimental results are illustrated by PDD and transverse dose profiles at $3 \mathrm{~cm}$ and $d_{\text {max }}$ depths in Fig. 2 for $8 \mathrm{~cm}$ of B0 and B30 bevel applicators, at $9 \mathrm{MeV}$ energy. Gamma index values are also presented. The model was considered validated as $98.27 \%$ of all evaluated points, for all energies, applicator diameters and bevel angles, verified gamma $\leqslant 1$.

The IORT numerical model was proved equally reliable in nonreference conditions with shielding, yielding $98.66 \%$ of evaluated points with gamma $\leqslant 1(2 \% / 2 \mathrm{~mm})$. Measured and simulated transverse dose profiles obtained at different depths are shown in Fig. 3, for the lateral insertion of two lead sheets of $2.4 \mathrm{~mm}$ total thickness.

\subsection{Studies of shielding efficiency}

\subsubsection{Dependency on lead thickness and beam energy}

The transverse dose profiles shown in Fig. 4 were obtained considering a 7B0 applicator, for different lead thicknesses and different beam energies. They allow the determination of the required lead thickness for efficient beam attenuation. Table 1 summarizes the estimated minimum lead thickness (min_Pb) for each beam energy. The results show a very good agreement with the data of Bagne et al. Despite this, it is important to emphasize the necessity of confirming the values of lead thickness for different IORT machines before clinical introduction of shielding [4].

For the case considered, where sheets were laterally inserted $1.6 \mathrm{~cm}$ in the radiation field, the PDD with shielding and the PDD with an air gap of the same thickness have a difference of $\pm 3 \%$, for every beam energy and a maximum shield thickness of $6.0 \mathrm{~mm}$ (data not shown). This agrees well with the data of Biggs et al. (2-3\% difference in the output factors) [5]. Combining the two effects (air gap plus shielding), the maximum clinical axis dose modification is $\pm 5 \%$.

\subsubsection{Effect of insufficient thickness}

When the lead thickness is insufficient, an additional lateral local dose increase may occur, as shown in Fig. 4. These hotspots are due to the perturbation of lateral electronic equilibrium by the presence of shielding materials. In the present study these

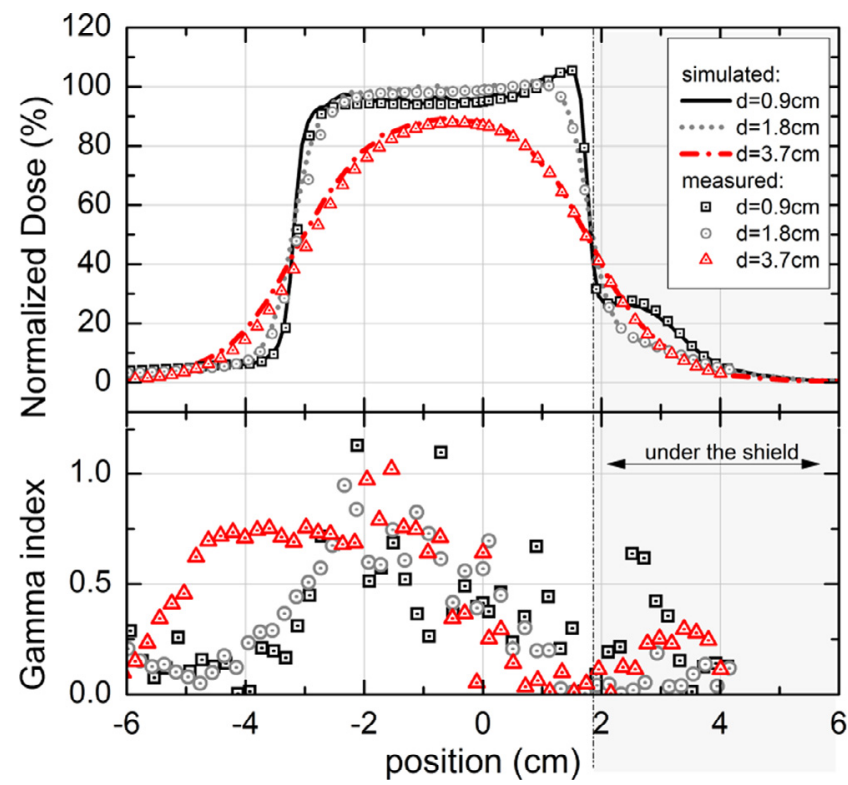

Fig. 3. Dose profiles obtained with 2 lead shielding sheets (total thickness $2.4 \mathrm{~mm}$ ) inserted at the border of the radiation field, at $12 \mathrm{MeV}$ beam energy, with a $7 \mathrm{~cm}$ diameter B0 applicator: (top) experimental and simulated data, and (bottom) corresponding gamma index analysis. 


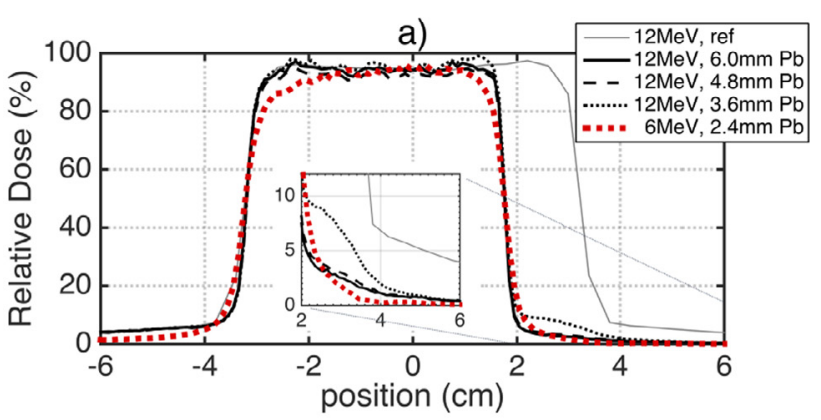

b)
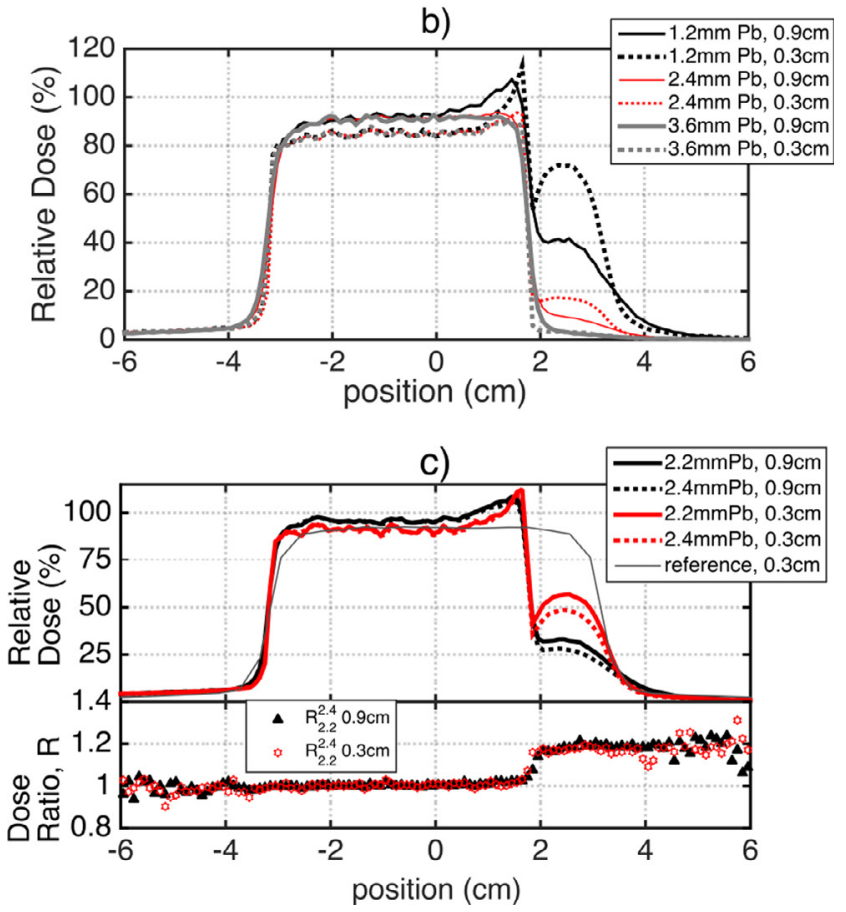

Fig. 4. Transverse dose profiles showing the effect of partial beam field shielding on a $7 \mathrm{~cm}$ B0 applicator, for different shield thickness: a) at $9 \mathrm{~mm}$ depth, for 6 and $12 \mathrm{MeV}$ IORT beam; b) at 3 and $9 \mathrm{~mm}$ depth, for a $9 \mathrm{MeV}$ beam; c) at 3 and $9 \mathrm{~mm}$ depth, with lead sheets of $2.2 \mathrm{~mm}$ and $2.4 \mathrm{~mm}$, for a $12 \mathrm{MeV}$ beam (top graph) - the bottom graph shows the ratio of doses $R_{2.2}^{2.4}$.

Table 1

Summary of obtained lead thickness required for IORT beam shielding.

\begin{tabular}{llll}
\hline & $6 \mathrm{MeV}$ & $9 \mathrm{MeV}$ & $12 \mathrm{MeV}$ \\
\hline Lead thickness [Bagne et al. [4]] & $2.4 \mathrm{~mm}$ & $4.0 \mathrm{~mm}$ & $4.8 \mathrm{~mm}$ \\
Required lead thickness (min_Pb) & $2.4 \mathrm{~mm}$ & $3.6 \mathrm{~mm}$ & $4.8 \mathrm{~mm}$ \\
Residual dose under the shield & $<4 \%$ & $<4 \%$ & $<4 \%$
\end{tabular}

hotspots were observed for different beam energies, and for different lead thicknesses. Lateral dose increases when less shielding is used, but it is still around $10 \%$ at $3 \mathrm{~mm}$ depth, for $2.4 \mathrm{~mm}$ lead thickness (2/3 of min_Pb) with $9 \mathrm{MeV}$ electron beam (Fig. 4b)).

Total lead thickness is dependent on the uncertainty of individual sheet thickness. This scenario was evaluated considering a variability of shielding sheet thickness of $0.1 \mathrm{~mm}$, resulting in a potential total reduction of $0.2 \mathrm{~mm}$ when 2 sheets are used. For a $12 \mathrm{MeV}$ beam and a 7B0 applicator, the simulated transverse dose profiles for lead thickness of $2.4 \mathrm{~mm}\left(1 / 2 \mathrm{~min} \_\mathrm{Pb}\right)$ and $2.2 \mathrm{~mm}$ are compared in Fig. 4c), at 3 and $9 \mathrm{~mm}$ depth in water phantom. The ratio between the profiles relative to the two shielding configurations, defined as $R_{2.2}^{2.4}=D(2.2) / D(2.4)$, is also shown in the same graph, for both depths. For the simulated scenario, a discrepancy between the actual and the nominal total lead thickness of $0.2 \mathrm{~mm}$ determines a relative increment of dose in the shielded region by $20 \%$ at both depths ( 3 and $9 \mathrm{~mm}$ ).

These two effects underscore the importance of using shielding with sufficient lead thickness.

\section{3. $2 D$ dose distributions}

To allow a better visualization of shielding effects, both laterally and in depth, transverse central plane dose distributions were extracted from 3D dose simulations. Representative examples of 2D dose maps are shown in Fig. 5 for $12 \mathrm{MeV}$ IORT configurations. Similar behavior was observed at 6 and $9 \mathrm{MeV}$, but $12 \mathrm{MeV}$ was chosen for presentation because the greater penetration depth allows a better visualization.

For comparison, a reference dose distribution of $12 \mathrm{MeV}$ and 7B0 applicator is shown in Fig. 5a). The 2D dose distribution resulting from the insertion of a $3.6 \mathrm{~mm}$ thick lead shielding $1.6 \mathrm{~cm}$ into the applicator is shown for a 7B0 applicator in Fig. 5c), and for 7B30 applicator in Fig. 5b) and (d). As bevels B30 and B45 are the most often used, the results for the B30 bevel with shielding are presented. The effect of B45 (not shown) is similar, but with more pronounced inclination, so the physical effects are superposed in space making visualization harder. The dose profiles corresponding to the vertical lines in Fig. 5, are shown in Fig. 6a), for several shielding thicknesses allowing a quantitative analysis of the dose, in depth. Following the expected dose decrease with depth, a recovery is observed until a relative maximum occurs. Both amplitude and location of the outbulge relative maximum depend on beam energy, but are independent of shielding thickness. For comparison, Table 2 summarizes the results on the location and relative dose maxima obtained from Fig. $6 \mathrm{~b}$ for the B0 configurations, at 6, 9 and $12 \mathrm{MeV}$ beams, for the cases of homogeneous phantoms. The lead thickness indicated in Table 2 is the threshold above which it is possible to distinguish the relative maximum.

\subsection{Bone influence on dose distribution}

Fig. 7 shows the dose distribution in depth, beneath the shield, in the middle position, between the shield edge and the external border of the B0 applicator, for a $9 \mathrm{MeV}$ beam, for the scenarios of a bone insertion similar to a pelvic IORT scenario and a homogeneous water phantom. The in-depth dose profiles show the same trend. Table 2 was complemented with data obtained for the same energies in the bone insertion configuration, for comparison. The values on Table 2 allow the estimation of expected clinical impact (with bone) as compared to the known reference conditions that can be measured in water.

\section{Discussion}

\subsection{Impact of shielding in IORT dose distributions}

\subsubsection{Surface shielding efficiency}

The amount of dose reduction under the shielding varies with depth. In the zoomed graph of Fig. 4a) the transverse profile for $7 \mathrm{B0} / 12 \mathrm{MeV}$ with $3.6 \mathrm{~mm}$ of lead (3/4 min_Pb), obtained at $0.9 \mathrm{~cm}$ depth, shows that the dose values are almost completely below $10 \%$ of Dmax. However, the vertical dose profiles shown in Fig. 6a), taken in the middle position of the beam shielded field, $8 \mathrm{~mm}$ away from the applicator border, show that for the same situation the dose at the surface may reach values around 30\% of Dmax. The residual surface dose increases rapidly with decreasing lead thickness: for $2.4 \mathrm{~mm}\left(1 / 2 \mathrm{~min} \_\mathrm{Pb}\right)$, it is still around $60 \%$ of Dmax for the same configuration (Fig. 6a). The same effect is 


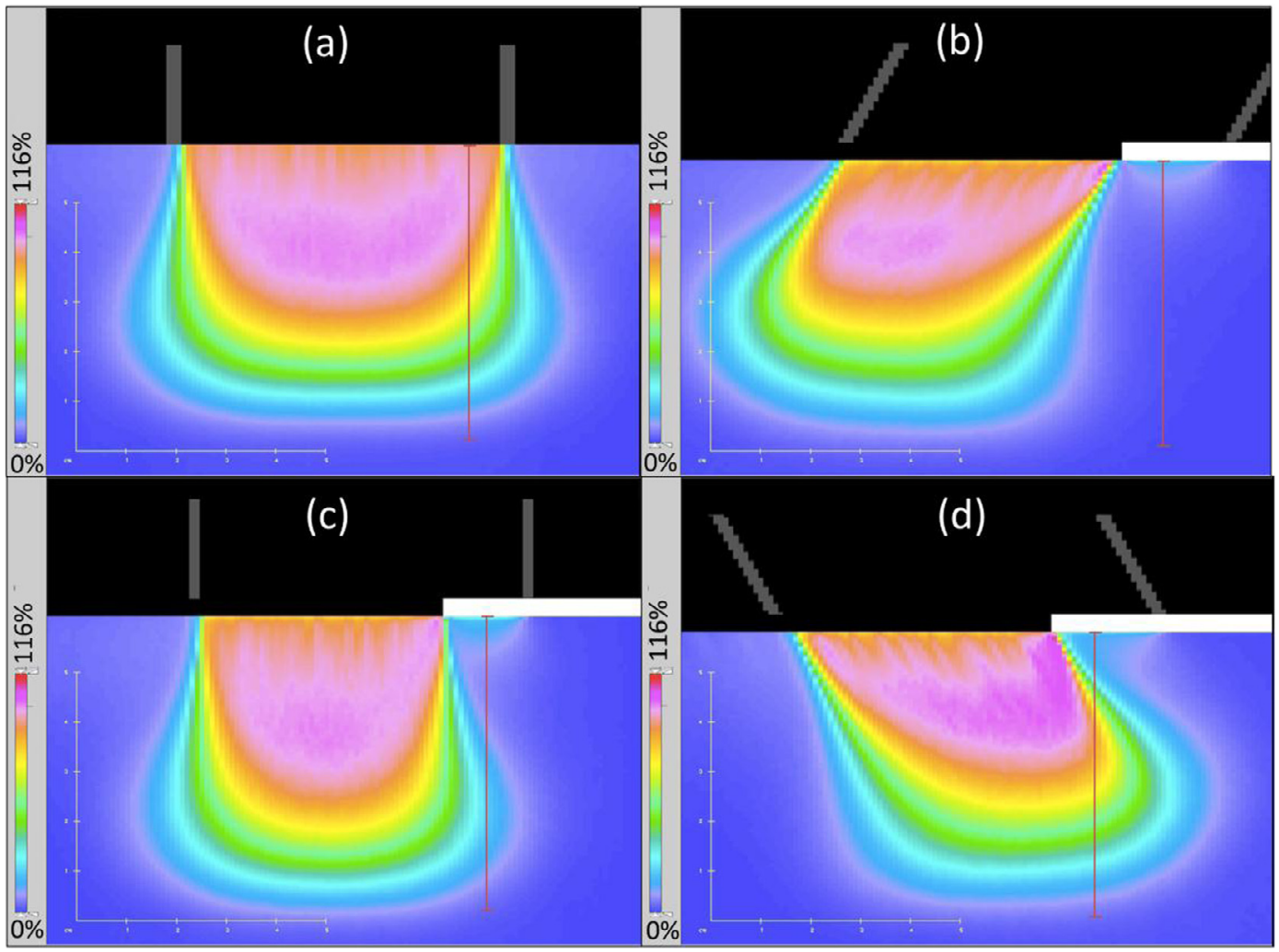

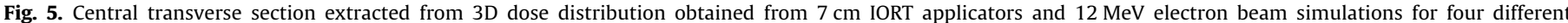

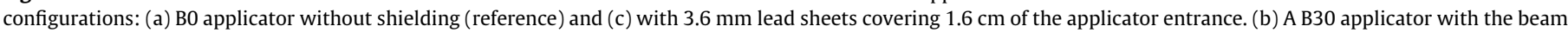
directed away and (d) towards the $3.6 \mathrm{~mm}$ lead sheets that are covering $1.9 \mathrm{~cm}$ of the applicator entrance.

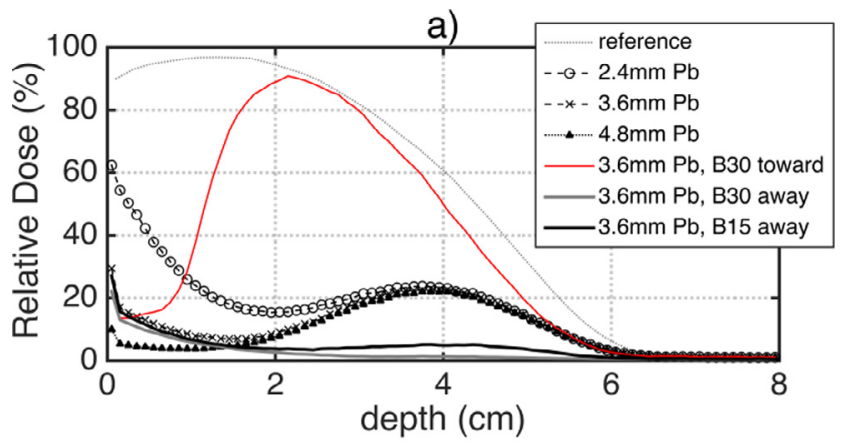

b)

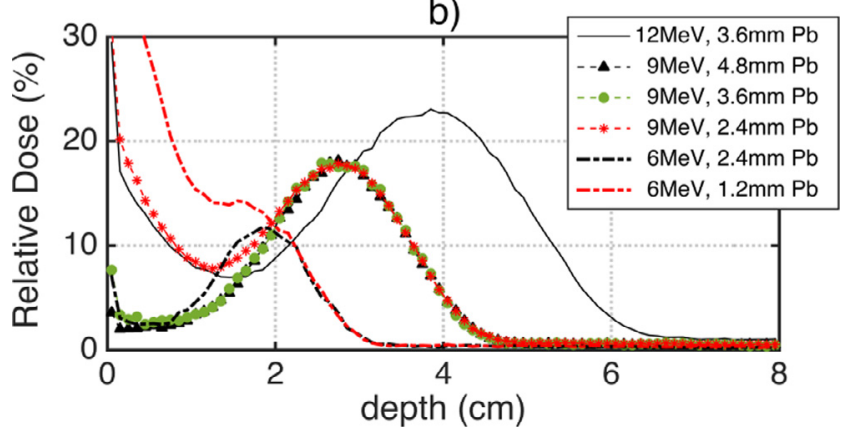

Fig. 6. Vertical dose profiles: a) at $12 \mathrm{MeV}$, below the shielding for the situations displayed in Fig. 5, using a $7 \mathrm{~cm}$ applicator and for other lead thicknesses $(6 \mathrm{~mm}$, $4.8 \mathrm{~mm}$, and $2.4 \mathrm{~mm}$ ) - the non-shielded (reference) profile is also displayed for comparison; b) for $6 \mathrm{MeV}, 9 \mathrm{MeV}$ and $12 \mathrm{MeV}$, in the same position, obtained for different lead thicknesses.

observed in Fig. 4b), where the transverse profiles obtained for 7B0/9 MeV $1.2 \mathrm{~mm}$ lead are markedly different at 0.3 and $0.9 \mathrm{~cm}$ depth.

\subsubsection{Deep shielding efficiency}

In pelvic IORT actual organs at risk may be located deeper in tissue. At greater depth, the choice of lead thickness does not seem to be relevant in terms of dose reduction. When an appropriate shielding thickness is applied, there is a visible dose reduction near the lead sheet. However as shown in Fig. 6a) for $12 \mathrm{MeV}$ and B0, at around $1.5 \mathrm{~cm}$ depth the dose values start to increase, reaching a considerable $22 \%$ of Dmax at $3.8 \mathrm{~cm}$ depth. The in-depth increase of dose is relatively independent of shielding thickness, as it is due to side-scattering events in the water phantom, broadening the shape of the electron beam profile. The broadening of the beam is also present at lower depths at the edge of an unshielded applicator, as seen on Fig. 5a). The magnitude of this effect is naturally linked to the pathlength of secondary electrons and its dependency on the beam energy.

In addition, both the depths and the percentage dose depend on the distance between the profile and the edge of the shielding. Under the shielding and at $1 \mathrm{~mm}$ from its edge, the $12 \mathrm{MeV}$ dose reaches $\approx 52 \%$ at approximately $2.6 \mathrm{~cm}$, and the $9 \mathrm{MeV}$ dose reaches $50 \%$ at $\approx 2.0 \mathrm{~cm}$.

\subsubsection{Shielding efficiency with beveled applicators}

Beveled applicators $\left(30^{\circ}\right.$ and $\left.45^{\circ}\right)$ are the most frequently used in pelvic IORT for rectal cancer [19]. When using beveled applicators there are two possible irradiation geometries, depending on whether the beam is directed away from the shielding, as shown in Fig. 5b), or towards it, as shown in Fig. 5d). When the beam is directed away from the shielding, the increased dose at further depths under the lead sheets will not be present (Fig. 5b)). This will ensure a better dose sparing to areas far beneath the shield, as shown in Fig. 6a), where the vertical dose profile reaches $5 \%$ and 3\% maximum for B15 and B30 beveled applicators respectively, at around $3.8 \mathrm{~cm}$ depth. As expected, for the opposite geometry 
Table 2

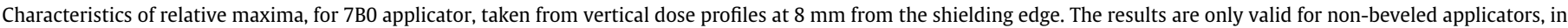
the specified configurations.

\begin{tabular}{|c|c|c|c|c|c|c|}
\hline \multirow[t]{2}{*}{ Beam energy (MeV) } & \multicolumn{3}{|c|}{ Water phantom } & \multicolumn{3}{|c|}{ Water phantom w/bone } \\
\hline & 6 & 9 & 12 & 6 & 9 & 12 \\
\hline Lead thickness (mm) & $>1.2$ & $>2.4$ & $>2.4$ & & & \\
\hline Maximum Amplitude & $12 \%$ & $18 \%$ & $22 \%$ & $4 \%$ & $8 \%$ & $14 \%$ \\
\hline Maximum location $(\mathrm{cm})$ & 1.9 & 2.8 & 3.8 & 1.1 & 1.6 & 2.2 \\
\hline
\end{tabular}

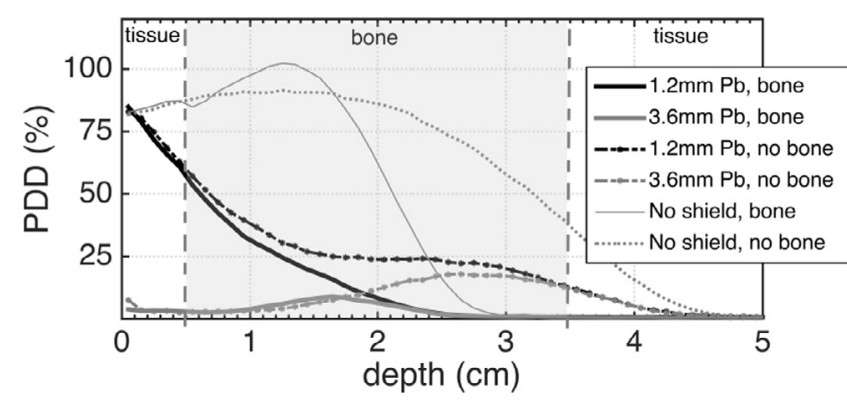

Fig. 7. Depth dose profiles profiles taken from the simulation of a partially shielded 7B0 applicator, at $9 \mathrm{MeV}$. The profiles are located under the shielding. Continuous line show the results for a homogeneous water phantom (none, 1.2 and $3.6 \mathrm{~mm} \mathrm{~Pb}$ shielding); dashed lines show the results for a water phantom with an insertion of a $3 \mathrm{~cm}$ bone layer at $5 \mathrm{~mm}$ depth (none, 1.2 and $3.6 \mathrm{~mm}$ Pb shielding).

(Fig. 5d)), the shielding only reduces the dose in the first $1.0 \mathrm{~cm}$ depth (for $12 \mathrm{MeV}$ ), as seen in Fig. 6a), because the beam is directed towards the region under the lead. For a B45 bevel the results are similar and more pronounced.

These results may seem obvious, but it is important for radiooncologists to have a chance to visualize them and to be aware of their impact on dose when deciding to shield organs at risk. Also, these results show that the effect is present even for a small bevel angle (B15). If the critical organ to be shielded is likely to extend 3$4 \mathrm{~cm}$ in depth, and the surface to be irradiated is such that both non-beveled and B15 applicators can be used, our results suggest that it could be advantageous to use a B15 applicator pointing in the appropriate direction, to increase the effect of the shielding. However, this gain in shielding effectiveness is obtained at the cost of extending the beam profile in the opposite direction. Moreover, in IORT, the treatment setup is often not completely free, due to geometrical and anatomical constraints.

\subsection{Effect of bone on shielded profiles}

The higher density of bone with respect to water produces sensible modification of the dose levels reached in depth, and in particular, beneath the shield, where dose reduction is intended.

As shown in Fig. 7, an initial decrease of dose is observed due to the shielding, then, a partial recover with a relative maximum due to scattering effects is observed. Different level and depth of the relative maximum is encountered when bone is considered (Table 2).

The higher density of the bone with respect to the water reduces the distance travelled by the secondary electrons, both in depth and laterally. Consequently, both the depth and the maximum dose level due to scattering effects are reduced with respect to the water homogeneous case correspondent dose profile, in the middle position between the shield edge and the external border.

Also, the results in Fig. 7 show that the presence of bone $5 \mathrm{~mm}$ below the surface produces very little backscatter, and has practically no effect on the unshielded surface dose. This is an interesting result for the interpretation of in vivo measurements in pelvic
IORT, and was also expected from published data. Martignano et al. simulated different high density shielding materials at $2 \mathrm{~cm}$ depth, and observed a strong reduction of backscattering for shielding materials of lower density, such as aluminum [10].

When a beveled applicator is used, and a bone layer is introduced as described above, the effects observed are similar to those obtained for non-beveled applicators. The dose depth profiles for B15 and B30 under the shield have the same trend as the respective profiles in homogeneous phantom case, slightly modified by the presence of the bone. Particularly, when B15 and B30 are considered, oriented in the direction away from the shield, the profiles show $2 \%$ as maximum. Moreover, if the beveled applicator is 180 deg rotated around the clinical axis, the dose values beneath the shield are considerably higher than those of the non-rotated configuration, even if lower and at smaller depth than the correspondent case in the water homogeneous phantom.

While our results were obtained for a particular LINAC, the effects observed should be qualitatively general, for electron beams with similar energies, although quantitative results such as optimal shield thickness may vary. In this work, only flat irradiation surfaces were considered. However, in the future, more realistic shielding scenarios may be considered as well, combining lead shielding with, for example, a concave irradiation surface with a curvature typical of the sacrum.

\section{Conclusions}

A set of IORT configurations was studied under the context of using shielding in pelvic IORT for rectal cancer, to provide quantitative information to assist radiation oncologists prescribing the therapy dose. An IORT machine based on a Varian CLINAC 2100CD was modeled with BEAMnrc and EGS++ codes and validated. Dose distributions resulting from the introduction of shielding elements within the IORT radiation field were evaluated, considering a homogeneous water phantom and a water phantom with an insertion of bone layer. From the presented results it was shown that: a considerable increase in dose in the lateral vicinity of the lead shielding sheet is associated with insufficient lead thickness; when sufficient shielding is used, no lateral hotspots occur and the shielding is effective near the surface; in depth, lateral scattering effects are observed and the lateral enlargement of the dose distribution is almost independent of lead thickness; this effect can be minimized by using a beveled applicator directed away from the shielding. The effect of having bone on the irradiation field was also studied. These results should be taken in consideration by radio-oncologists when choosing the best lead thickness, shape and position.

\section{Acknowledgements}

The authors are grateful to O. Sousa for useful discussions. This work is financed by National Funds through FCT - Fundação para a Ciência e a Tecnologia in the scope of the project PTDC/SAUENB/117631/2010, by the Programa Operacional Regional do Norte de Portugal (ON.2 - O Novo Norte) in the scope of the project " $\mathrm{N}$ 
ORTE-07-0124-FEDER-000058", and by ERDF - European Regional Development Fund through the COMPETE Programme in the scope of the project "reference FCOMP-01-0124-FEDER-021141".

\section{Appendix A. Supplementary data}

Supplementary data associated with this article can be found, in the online version, at http://dx.doi.org/10.1016/j.ejmp.2016.10. 004.

\section{References}

[1] Calvo F, Meirino RM, Orecchia R. Intraoperative radiation therapy first part: rationale and techniques. Crit Rev Oncol Hematol 2006;59:106-15. http://dx. doi.org/10.1016/j.critrevonc.2005.11.004.

[2] Palta JR, Biggs PJ, Hazle JD, Huq MS, Dahl RA, Ochran TG et al. Intraoperative electron beam radiation therapy: technique, dosimetry, and dose specification: report of task force 48 of the radiation therapy committee, american association of physicists in medicine. Int $\mathrm{J}$ Radiat Oncol Biol Phys 1995:33:725-46.

[3] Beddar AS, Krishnan S. Intraoperative radiotherapy using a mobile electron LINAC: a retroperitoneal sarcoma case. J Appl Clin Med Phys 2005;6:95-107.

[4] Bagne FR, Ralph R, Dobelbower Jr AJM, Bronng DG. Treatment of cancer of the pancreas by intraoperative electron beam therapy: physical and biological aspects. Int J Radiat Oncol 1989;16:231-42.

[5] Biggs PJ, Epp ER, Ling CC, Novack DH, Michaels HB. Dosimetry, field shaping and other considerations for intra-operative electron therapy. Int J Radiat Oncol 1981.

[6] Pascau J, Ph D, Santos A, Morillo V, Calvo FA, Bouche A, et al. An innovative tool for intraoperative electron beam radiotherapy simulation and planning : description and initial evaluation by radiation oncologists. Int J Radiat Oncol Biol Phys 2012;83:287-95. http://dx.doi.org/10.1016/j.ijrobp.2011.12.063.

[7] Costa F, Sarmento S, Sousa O. Assessment of clinically relevant dose distributions in pelvic IOERT using Gafchromic EBT3 films. Phys Med 2015;31:692-701. http://dx.doi.org/10.1016/j.ejmp.2015.05.013.
[8] Catalano M, Agosteo S, Moretti R, Andreoli S. Montecarlo simulation code in optimisation of the IntraOperative Radiation Therapy treatment with mobile dedicated accelerator. J Phys: Conf Ser 2007;74(74). http://dx.doi.org/10.1088/ 1742-6596/74/1/012002. 11.

[9] Neto AJ, de C, Haddad CMK, Pelosi EL. Monte carlo simulation as an auxiliary tool for electron beam quality specification for intra-operative radiotherapy. Braz J Phys 2005;35:1-4.

[10] Martignano A, Menegotti L, Valentini A. Monte Carlo investigation of breast intraoperative radiation therapy with metal attenuator plates. Med Phys 2007;34:4578. http://dx.doi.org/10.1118/1.2805089.

[11] Oshima T, Aoyama Y, Shimozato T, Sawaki M, Imai T, Ito Y, et al. An experimental attenuation plate to improve the dose distribution in intraoperative electron beam radiotherapy for breast cancer. Phys Med Biol 2009;54:3491-500. http://dx.doi.org/10.1088/0031-9155/54/11/014.

[12] Soriani A, Iaccarino G, Felici G, Ciccotelli A, Pinnaro P, Giordano C, et al Development and optimization of a beam shaper device for a mobile dedicated IOERT accelerator. Med Phys 2012;39:6080-9. http://dx.doi.org/10.1118 1.4749968.

[13] Rogers DWO, Walters B, Kawrakow I BEAMnrc users manual Nationa Research Council of Canada report PIRS-0509(A)revL, vol. 509, 2005. Ottawa.

[14] Walters B, Kawrakow I, Rogers DWO. DOSXYZnrc users manual National Research Council of Canada, report PIRS-794revB, 2009. Ottawa.

[15] Kawrakow I. egspp: the EGSnrc C++ class library National Research Council of Canada report PIRS-899, 2005.

[16] Iaccarino G, Strigari L, D’Andrea M, Bellesi L, Felici G, Ciccotelli A, et al. Monte Carlo simulation of electron beams generated by a $12 \mathrm{MeV}$ dedicated mobile IORT accelerator. Phys Med Biol 2011;56:4579-96. http://dx.doi.org/10.1088 0031-9155/56/14/022.

[17] Low DA, Harms WB, Mutic S, Purdy JA. A technique for the quantitative evaluation of dose distributions. Med Phys 1998;25:656-61.

[18] Deasy JO, Blanco AI, Clark VH. CERR: a computational environment for radiotherapy research. Med Phys 2003;30:979-85. http://dx.doi.org/10.1118 1.1568978.

[19] Krengli M, Sedlmayer F, Calvo FA, Sperk E, Pisani C, Sole CV, Fastner G Gonzalez C, Wenz F. ISIORT pooled analysis 2013 update: clinical and technical characteristics of intraoperative radiotherapy. Transl Cancer Res 2014;3 (1):48-58. http://dx.doi.org/10.3978/i.issn.2218-676X.2014.01.02 